NSF-ITP-99-135

\title{
S-duality and Tensionless 5-branes in Compactified Heterotic String Theory
}

\author{
Morten Krogh \\ Institute for Theoretical Physics \\ University of California, Santa Barbara, CA 93106 \\ USA \\ E-mail: krogh@itp.ucsb.edu
}

\begin{abstract}
We give a simple proof of the known S-duality of Heterotic String theory compactified on a $\mathbf{T}^{6}$. Using this S-duality we calculate the tensions for a class of BPS 5-branes in Heterotic String theory on a $\mathbf{S}^{1}$. One of these, the Kaluza-Klein monopole, becomes tensionless when the radius of the $\mathbf{S}^{1}$ is equal to the string length. We study the question of stability of the Heterotic NS5-brane with a transverse circle. For large radii the NS5-brane is absolutely stable. However for small radii it is only marginally stable. We also study the moduli space of 2 Kaluza-Klein monopoles and show that it is equal to the moduli space of a Heterotic $A_{1}$ singularity.
\end{abstract}

November 1999 


\section{Introduction}

In this paper we study various aspects of Heterotic string theory [ [1]]. Heterotic string theory compactified on $\mathbf{T}^{6}$ has a non-perturbative $S L(2, \mathbf{Z})$ duality. The duality was first presented by Font, Ibanez, Lust and Quevedo in [a] Evidence in favor of it was presented by A. Sen in [3]. Later the duality was proven [ [on $\mathbf{T}^{4}$ [5]. Here we present a simple proof of this strong-weak coupling duality using only the fact that M-theory on a circle is type IIA and T-dualities in type II.

We explore the consequences of this duality for Heterotic string theory on a circle with unbroken gauge group. We find an exact formula for the tension of the Kaluza-Klein monopole. The result is that the tension is

$$
T=\left|\frac{m_{s}^{6}}{\lambda^{2}}-\frac{m_{s}^{8} R^{2}}{\lambda^{2}}\right|
$$

where $m_{s}$ is the string mass, $R$ is the radius of the circle and $\lambda$ is the Heterotic coupling constant. We see that at the selfdual radius, $m_{s} R=1$, this 5 brane becomes tensionless. This has some equivalent statements in some dual systems. For instance compactifying this further on a $\mathbf{T}^{3}$ this system is dual to type IIA on $K 3$ with a $D 6$-brane wrapped on the $K 3$. The statement here is that the resulting 2-brane becomes tensionless for a certain value of the volume of the $K 3$.

We also study the stability of a Heterotic NS5-brane with a transverse circle. It turns out that for large radii the NS5-brane is completely stable, but for radii smaller than the selfdual radius the NS5-brane is only marginally stable. It can split into two 5-branes, whose sum of tensions is equal to the NS5-brane tension.

Finally we discuss the moduli space of two Kaluza-Klein monopoles. The moduli space of ADE singularities has recently been studied [6, 0, 8, 9]. In the case of an $A_{1}$ singularity the moduli space was found to be the Atiyah-Hitchin 
space. We show explicitly following the methods of [6] that the moduli space of two Kaluza-Klein monopoles is still the Atiyah-Hitchin space. In other words the transverse circle does not change the moduli space.

The organisation of the paper is as follows. In section(1) we explain and prove the strong-weak coupling duality of 4-dimensional Heterotic theory. In section(2) we study a certain class of 5-branes of the Heterotic theory on a circle and find the tensions for those 5 -branes which are BPS. We find that a certain 5-brane becomes tensionless when the radius of the compactification circle is selfdual under T-duality. In section(3) we identify this 5-brane as the Taub-Nut solution, also called the Kaluza-Klein monopole. In section(4) we study the question of stability of the NS5-brane in 9-dimensional Heterotic theory. In section(5) we study the moduli space of two Kaluza-Klein monopoles.

\section{Proof of SL(2,Z) duality}

Heterotic string theory compactified on a $\mathbf{T}^{6}$ is known to have a moduli space which is

$$
\begin{aligned}
& (O(22, \mathbf{R}) \times O(6, \mathbf{R})) \backslash O(22,6, \mathbf{R}) / O(22,6, \mathbf{Z}) \times \\
& S O(2, \mathbf{R}) \backslash S L(2, \mathbf{R}) / S L(2, \mathbf{Z})
\end{aligned}
$$

The U-duality group is

$$
O(22,6, \mathbf{Z}) \times S L(2, \mathbf{Z})
$$

The $O(22,6, \mathbf{Z})$ is the perturbative T-duality group. The $S L(2, \mathbf{Z})$ is a nonperturbative duality. Evidence in favor of it was presented by A. Sen in [3]. The evidence consisted of four pieces:

- The low energy equations of motion had the symmetry.

- The allowed spectrum of electric and magnetic charges was consistent with the duality. 
- Agreement of certain BPS masses in 4 dimensions.

- Certain Yukawa couplings of BPS saturated fields could be checked explicitly.

Later the duality was proven [4] by relating it to the duality between Type IIA on K3 and Heterotic on $\mathbf{T}^{4}$ [5]. By compactifying this duality further on a $\mathbf{T}^{2}$ the non perturbative $S L(2, \mathbf{Z})$ on the Heterotic side could be identified with one of the $S L(2, \mathbf{Z})$ factors in the T-duality of the $\mathbf{T}^{2}$ on the Type IIA side.

The aim of this section is to prove the existence of this $S L(2, \mathbf{Z})$ duality in the Heterotic theory on $\mathbf{T}^{6}$ in a simpler way.

The two different Heterotic theories in 10 dimensions are dual when compactified on a circle and certainly also on a $\mathbf{T}^{6}$, so we do not have to distinguish between the two. The moduli of the compactification can be parametrised as follows. There is the metric on $\mathbf{T}^{6}$, the $B$-field on $\mathbf{T}^{6}$, $E_{8} \times E_{8}$ Wilson lines on the 6 circles and the 4 dimensional string coupling, $\lambda$. Furthermore, and of central importance to this $S L(2, \mathbf{Z})$ duality, there is a two-form in 4 dimensions coming from the NS-NS $B$-field in 10 dimensions. In 4 dimensions a two-form is dual to a scalar. Hence we get another scalar, $a$, called the axion. Another way of understanding the scalar $a$ is to dualise the $B$-field in ten dimensions to a six-form $B^{(6)}$. The integral of this six-form on $\mathbf{T}^{6}$ gives a scalar in 4 dimensions. We can conveniently collect the string coupling, $\lambda$, and the axion into a complex number in the upper halfplane,

$$
\tau=a+i \frac{1}{\lambda}
$$

The $S L(2, \mathbf{Z})$ duality acts as follows. It leaves the metric, the $B^{2}$-field on $\mathbf{T}^{6}$ and the Wilson lines invariant and it acts on $\tau$ as

$$
\tau \rightarrow \frac{p \tau+q}{r \tau+s}
$$


where

$$
\left(\begin{array}{ll}
p & q \\
r & s
\end{array}\right) \in S L(2, \mathbf{Z})
$$

It is well known that $\mathrm{SL}(2, \mathbf{Z})$ is generated by the two elements

$$
S=\left(\begin{array}{cc}
0 & -1 \\
1 & 0
\end{array}\right)
$$

and

$$
T=\left(\begin{array}{ll}
1 & 1 \\
0 & 1
\end{array}\right)
$$

To show the full $S L(2, \mathbf{Z})$ duality we thus just have to show that $S$ and $T$ are dualities. $T$ is actually still perturbative, since it it does not touch the coupling. $T$ shifts the axion. This is a symmetry as follows from the gauge symmetry of the six-form, $B^{(6)}$ in ten dimensions. In four dimensions there are instantons coming from Euclidean NS5-branes wrapped on $\mathbf{T}^{6}$. They are weighted with $e^{i 2 \pi a}$ in the action, showing that $a \rightarrow a+1$ is a symmetry. It is similar to the well known $2 \pi$ shift of the $\theta$-angle in 4 dimensional gauge theories.

The non trivial point is to establish existence of the strong-weak duality, $S$. To do that we will use the Horava-Witten picture of $E_{8} \times E_{8}$ Heterotic string theory [10]. By compactifying further on a $\mathbf{T}^{6}$ we see that the Heterotic string on $\mathbf{T}^{6}$ can be represented as M-theory on $\mathbf{T}^{7}$ modded out by a $\mathbf{Z}_{2}$ symmetry. To be more precise, consider M-theory on $\mathbf{R}^{1,3} \times \mathbf{T}^{7}$. The coordinates on $\mathbf{T}^{7}$ are called $x^{5}, x^{6}, \ldots, x^{11}$. M-theory has a 3-form field, $C^{(3)}$. The $\mathbf{Z}_{2}$ group is generated by an element $\omega$ which acts as follows. $\omega$ is a parity operation on $x^{11}$ and changes the sign of $C^{(3)}$. The parity operation alone is not a symmetry of M-theory because of the Chern-Simons term in 11-dimensional supergravity. We will now perform a certain U-duality transformation, say $\mathrm{U}$, of M-theory on $\mathbf{T}^{7}$. Then we will obtain M-theory on another $\mathbf{T}^{7}$. The symmetry $\omega$ will be mapped to another symmetry

$$
\tilde{\omega}=U \omega U^{-1}
$$


We now mod out by the respective symmetry on both sides and identify the result. In other words M-theory on $\mathbf{T}^{7}$ modded out by $\omega$ is dual to M-theory on the dual $\mathbf{T}^{7}$ modded out by $\tilde{\omega}$.

We will now define the relevant U-duality transformation and track the transformation of the fields. We start with M-theory on $\mathbf{T}^{7}$ with radii $R_{5}, R_{6}, \ldots, R_{11}$. The 11-dimensional Planck Mass is called $M_{P}$. We will take the torus to be rectangular for simplicity. The chain of dualities is as follows.

- $(1)$

Go to Type IIA by using the 5 'th circle as the compactification circle. The string mass, $m_{s}$, coupling, $\lambda$ and radii, $r_{I}$ are

$$
\begin{aligned}
m_{s} & =\left(M_{P}^{3} R_{5}\right)^{\frac{1}{2}} \\
\lambda & =\left(M_{P} R_{5}\right)^{\frac{3}{2}} \\
r_{I} & =R_{I}, \quad I=6,7,8,9,10,11
\end{aligned}
$$

- $(2)$

Perform T-duality on the 5 circles with radii $R_{6}, \ldots, R_{10}$ to get to type IIB with parameters

$$
\begin{aligned}
m_{s} & =\left(M_{P}^{3} R_{5}\right)^{\frac{1}{2}} \\
\lambda & =\frac{1}{M_{P}^{6} R_{5} R_{6} R_{7} R_{8} R_{9} R_{10}} \\
r_{I} & =\frac{1}{M_{P}^{3} R_{5} R_{I}}, \quad I=6,7,8,9,10 \\
r_{11} & =R_{11}
\end{aligned}
$$

- $(3)$

Next we perform an S-duality of type IIB. The parameters become

$$
m_{s}=M_{P}^{\frac{9}{2}} R_{5}\left(R_{6} R_{7} R_{8} R_{9} R_{1} 0\right)^{\frac{1}{2}}
$$




$$
\begin{aligned}
\lambda & =M_{P}^{6} R_{5} R_{6} R_{7} R_{8} R_{9} R_{10} \\
r_{I} & =\frac{1}{M_{P}^{3} R_{5} R_{I}}, \quad I=6,7,8,9,10 \\
r_{11} & =R_{11}
\end{aligned}
$$

- (4)

This is followed by another T-duality on the 5 circles with radii $R_{6}, \ldots, R_{10}$ which brings us back to type IIA with parameters

$$
\begin{aligned}
m_{s} & =M_{P}^{\frac{9}{2}} R_{5}\left(R_{6} R_{7} R_{8} R_{9} R_{1} 0\right)^{\frac{1}{2}} \\
\lambda & =\frac{R_{5}}{M_{P}^{\frac{3}{2}}\left(R_{6} R_{7} R_{8} R_{9} R_{10}\right)^{\frac{1}{2}}} \\
r_{I} & =\frac{R_{I}}{M_{P}^{6} R_{5} R_{6} R_{7} R_{8} R_{9} R_{10}}, \quad I=6,7,8,9,10 \\
r_{11} & =R_{11}
\end{aligned}
$$

- (5)

Finally we lift this up to M-theory by opening up a circle which is called the 5 'th circle again. We arrive at M-theory on $\mathbf{T}^{7}$ with parameters

$$
\begin{aligned}
\tilde{M}_{P} & =M_{P}^{5}\left(R_{5} R_{6} R_{7} R_{8} R_{9} R_{10}\right)^{\frac{2}{3}} \\
\tilde{R}_{I} & =\frac{R_{I}}{M_{P}^{6} R_{5} R_{6} R_{7} R_{8} R_{9} R_{10}}, \quad I=5,6,7,8,9,10 \\
\tilde{R}_{11} & =R_{11}
\end{aligned}
$$

We now have two different $\mathbf{T}^{7}$ compactifications of M-theory which are dual. Actually the eleventh circle was not touched at all in this chain of dualities, so the duality is already a duality of M-theory on $\mathbf{T}^{6}$. We now want to $\bmod$ out by respectively $\omega$ and $\tilde{\omega}$ and identify the resulting theories. To do that we need to find $\tilde{\omega}$. In order to do that we must track the various fields of M-theory under the duality. The bosonic fields are the metric $g_{I J}$ and the 3form $C_{I J K}$ and the dual 6-form $C_{I J K L M N}$. The 3-form and 6-form are related 
but it is still an advantage to keep track of both as we will see below. Under $\omega$ they transform as

$$
\begin{aligned}
& g_{I J} \rightarrow g_{I J} \quad I, J=1, \ldots, 10 \\
& g_{I(11)} \rightarrow-g_{I(11)} \quad I=1, \ldots, 10 \\
& C_{I J K} \rightarrow-C_{I J K} \quad I, J, K=1, \ldots, 10 \\
& C_{I J(11)} \rightarrow C_{I J(11)} \quad I, J=1, \ldots, 10 \\
& C_{I J K L M N} \rightarrow C_{I J K L M N} \quad I, J, K, L, M, N=1, \ldots, 10 \\
& C_{I J K L M(11)} \rightarrow-C_{I J K L M(11)} \quad I, J, K, L, M=1, \ldots, 10
\end{aligned}
$$

The transformation law of the 6-form follows from the relation

$$
d C^{(3)}=* d C^{(6)}
$$

We now claim that under the U-duality transformation a field that transforms with eigenvalue 1 is mapped into another field with eigenvalue 1 and the same is true for eigenvalue -1 . To see that one has to track all the different fields through the chain of dualities. Let us here just do it explicitly for one case, namely $g_{1(10)}$. In the first step above it remains $g_{1(10)}$. In the second step it becomes $B_{1(10)}^{N S}$. In the third step it becomes $B_{1(10)}^{R R}$. In the 4 th step it becomes $C_{16789}^{R R}$. In the 5 th step it becomes $C_{156789}$. It is indeed true that both $g_{1(10)}$ and $C_{156789}$ have the same eigenvalue, namely 1.

This implies that $\tilde{\omega}=\omega$. We conclude that by modding out on both sides by $\omega$ we get a duality between two Heterotic theories on $\mathbf{T}^{6}$. In the Horava-Witten picture the relation between the M-theory parameters, $M_{P}$ and the radius of the eleventh circle $R_{11}$, and the Heterotic parameters, $m_{s}$ and $\lambda$ are

$$
\begin{aligned}
m_{s}^{2} & =M_{P}^{3} R \\
\lambda & =\left(M_{P} R\right)^{\frac{3}{2}}
\end{aligned}
$$


Using these relations and Eq.(5) we can relate the parameters of the Heterotic theory on one side of the duality with those on the other side.

$$
\begin{aligned}
\tilde{m}_{s} & =\frac{m_{s}^{7} R_{5} R_{6} R_{7} R_{8} R_{9} R_{10}}{\lambda^{2}} \\
\tilde{\lambda} & =\frac{m_{s}^{6} R_{5} R_{6} R_{7} R_{8} R_{9} R_{10}}{\lambda} \\
\tilde{R}_{I} & =\frac{\lambda^{2} R_{I}}{m_{s}^{6} R_{5} R_{6} R_{7} R_{8} R_{9} R_{10}} \quad I=5,6,7,8,9,10
\end{aligned}
$$

This looks a bit messy but we can understand it better by expressing it in the right variables. In the moduli space

$$
\begin{aligned}
& (O(22, \mathbf{R}) \times O(6, \mathbf{R})) \backslash O(22,6, \mathbf{R}) / O(22,6, \mathbf{Z}) \times \\
& S O(2, \mathbf{R}) \backslash S L(2, \mathbf{R}) / S L(2, \mathbf{Z})
\end{aligned}
$$

the radii of the torus are contained in the first factor and they are expressed in string units. We easily calculate that

$$
\tilde{m}_{2} \tilde{R}_{I}=m_{s} R_{I} \quad I=5,6,7,8,9,10
$$

This shows that the radii are unchanged in the right units. We could have worked with a non-rectangular torus if we wanted to. Since the rectangular torus is unchanged it is clear that any torus would be left unchanged.

The second factor of the moduli space is a complex number in the upper halfplane, $\tau$. The imaginary part of $\tau$ is $\frac{1}{\lambda_{4}}$ where $\lambda_{4}$ is the four-dimensional string coupling. The relation between the four-dimensional and ten dimensional coupling is

$$
\frac{1}{\lambda_{4}^{2}}=\frac{m_{s}^{6} R_{5} R_{6} R_{7} R_{8} R_{9} R_{10}}{\lambda^{2}}
$$

Using Eq.(6) we find that

$$
\tilde{\lambda}_{4}=\frac{1}{\lambda_{4}}
$$

This derivation was done with zero axion, and hence is exactly the element, $S$, of $S L(2, \mathbf{Z})$. We have now derived the existence of this duality only for a 
subset of the whole moduli space. However the existence of a duality in just one point implies that it extends to the whole moduli space. Its action is to leave the point in the first factor invariant and act as

$$
\tau \rightarrow \frac{-1}{\tau}
$$

on the second factor. In particular the Wilson lines of $E_{8} \times E_{8}$ are left invariant. In this derivation the Wilson lines could not be tracked since they only exist after modding out. This finishes our proof of the strong-weak duality. In the next section we will study some consequences of it.

\section{Tensionless 5-branes in Heterotic string the- ory on a circle}

In this section we will study the Heterotic theory on a circle of radius $R$. The gauge group could be either $E_{8} \times E_{8}$ or $\frac{\operatorname{Spin}(32)}{\mathbf{Z}_{2}}$. For simplicity we take the Wilosn line on the circle to be zero so the gauge group is unbroken. There are two $U(1)$ gauge fields in 9 dimensions, namely $g_{\mu 10}$ and $B_{\mu 10}$. The charges under these are respectively momentum amd winding. In perturbative string theory one can calculate the mass of ligthest particle with momentum $m$ and winding $n$. However it is only for certain $m$ and $n$ that the state is BPS. In the BPS case the mass calculated at string tree-level is exact. The quantum numbers and masses in the BPS cases are [1] :

- $(1)$

$$
m \geq 0, n \leq 0, \quad \text { or } \quad m \leq 0, n \geq 0, \quad M=\left|\frac{m}{R}-n m_{s}^{2} R\right|
$$

- $(2)$

$$
m=1, n=1, \quad \text { or } \quad m=-1, n=-1, \quad M=\left|\frac{1}{R}-m_{s}^{2} R\right|
$$


These conditions secure that there are no excited rightmoving oscillators. The last two states become massless at the selfdual radius. They are the $\mathrm{W}$ bosons of the enhanced $S U(2)$ gauge symmetry. By compactifying further on a $\mathbf{T}^{5}$ these particles still exist with the same masses. We can now apply the S-duality transformation discussed in the previous section. Let the radii of the $\mathbf{T}^{5}$ be $R_{5}, R_{6}, R_{7}, R_{8}, R_{9}$. The S-dual object of the particle with quantum numbers $(m, n)$ is a particle with a mass which is easily calculated to be.

$$
M=\left|m \frac{m_{s}^{6}}{\lambda^{2}}-n \frac{m_{s}^{8} R^{2}}{\lambda^{2}}\right| R_{5} R_{6} R_{7} R_{8} R_{9}
$$

We see that it is a particle with a mass proportional to the volume of the $\mathbf{T}^{5}$. This implies that it is a 5-brane in 9 dimensions with a tension of

$$
T_{(m, n)}=\left|m \frac{m_{s}^{6}}{\lambda^{2}}-n \frac{m_{s}^{8} R^{2}}{\lambda^{2}}\right|
$$

These 5-branes are magnetically charged under the two $U(1)$ gauge fields in 9 dimensions. The quantum number $m$ denotes NS5-brane charge. An NS5-brane indeed has tension

$$
T_{(1,0)}=\frac{m_{s}^{6}}{\lambda^{2}}
$$

The quantum number $n$ denotes the Kaluza-Klein monopole charge. In other words the circle of the compactification can be in a non-trivial bundle over the transverse $\mathbf{R}^{3}$ at infinity. $n$ measures the first chern class of this bundle. The 5-branes are BPS whenever the dual particles are. Let us look closer at one of the special cases listed above, $m=1, n=1$. The other one $m=-1, n=-1$ is just the antibrane. This BPS 5-brane has a tension which is

$$
T_{(1,1)}=\left|\frac{m_{s}^{6}}{\lambda^{2}}-\frac{m_{s}^{8} R^{2}}{\lambda^{2}}\right|
$$

We see that for large $R$ it is heavy but for the selfdual radius $m_{s} R=1$ it is tensionless. What is this 5-brane? In the next section we will see that it is the Taub-Nut space solution. 
Let us see how the two kinds of branes transform under T-duality. Heterotic string theory with gauge group, $\mathrm{G}$, on a circle without a Wilson line has a T-duality that preserves the gauge group. The relation between the original parameters and the new ones is

$$
\begin{aligned}
R^{\prime} & =\frac{1}{m_{s}^{2} R} \\
\lambda^{\prime} & =\frac{\lambda}{m_{s} R}
\end{aligned}
$$

with the same $m_{s}$. There is a gauge field, $g_{\mu 10}$, in 9 dimensions coming from the metric and a gauge field, $B_{\mu 10}$, coming from the $B$-field. The electric charge for $g_{\mu 10}$ is momentum around the circle. The magnetic charge is Kaluza-Klein monopole charge. The electric charge for $B_{\mu 10}$ is string winding and the magnetic charge is NS5-brane charge. These two gauge fields are swapped under the T-duality. Momentum and winding are swapped and Kaluza-Klein and NS5-brane charges are swapped. Under T-duality of the $\mathbf{S}^{1}$ the quantum numbers $m, n$ are mapped as follows

$$
m \rightarrow-n, \quad n \rightarrow-m
$$

Especially the Taub-Nut space is mapped to the Taub-Nut space with opposite orientation.

$$
(m, n)=(1,1) \rightarrow(m, n)=(-1,-1)
$$

\section{The Taub-Nut solution in the Heterotic String theory on a circle}

In this section we will discuss the supergravity solution for the Taub-Nut 5brane in the Heterotic string theory compactified on a circle down to 9 dimensions. Supergravity solutions for monopoles in compactified Heterotic string theories have been discussed extensively in the litterature [12, 13, 14, 15]. 
We review parts of the construction in order to understand that the brane with charges $(1,1)$ is the Taub-Nut 5 -brane. The gauge group could be either $E_{8} \times E_{8}$ or $\frac{\operatorname{Spin}(32)}{\mathbf{Z}_{2}}$. It will not make a difference for our purpose. We will take no Wilson line on the circle and hence keep the gauge group unbroken. By 5-brane we understand an object with $5+1$ dimensional Lorentz invariance and localised in the 3 transverse directions. There are two basic types of these, namely the NS5-brane and the Kaluza-Klein monopole. Here we only discuss the Kaluza-Klein monopole also called Taub-Nut space. The NS5-brane exists already in 10 dimensions and the 9 dimensional one is trivially obtained from the 10 dimensional one. 5-branes in 10 dimensions were discussed in [16]. The Kaluza-Klein monopole, on the other hand, only exists after compactification. Now we will discuss the supergravity solution of this object closely following the discussion of [16]. As we will see below the Kaluza-Klein monopole preserves half of the supersymmetry, i.e. 8 supercharges.

The field content of the Heterotic string theory in 10 dimensions is the metric, $\gamma_{M N}$, the dilaton, $\phi$, a two-form potential, $B$, a gauge potential, $A$, a gaugino $\chi$, a dilatino, $\lambda$ and a gravitino, $\psi_{M}$. The bosonic part of the action in 10 dimensions reads

$$
S=\frac{1}{2 \kappa^{2}} \int d^{10} x \sqrt{-g} e^{-2 \phi}\left(R+4(\nabla \phi)^{2}-\frac{1}{3} H^{2}-\frac{\alpha^{\prime}}{30} \operatorname{Tr} F^{2}\right)
$$

where the three-form antisymmetric tensor field strength is related to the two-form potential by the familiar anomaly equation [17].

$$
H=d B+\alpha^{\prime}\left(\omega_{3}^{L}(\Omega)-\frac{1}{30} \omega_{3}^{Y M}(A)\right)
$$

where $\omega_{3}$ is the Chern-Simons three-form. This equation implies

$$
d H=\alpha^{\prime}\left(\operatorname{tr} R \wedge R-\frac{1}{30} \operatorname{Tr} F \wedge F\right)
$$

The trace $T r$ is taken in the adjoint representation of the gauge group. The connection $\Omega$ appearing in Eq.(17) is a non-Riemannian connection related 
to the usual spin connection $\omega$ by

$$
\Omega_{M}^{A B}=\omega_{M}^{A B}-H_{M}^{A B}
$$

In these equations $M, N$ are Einstein indices and $A, B$ are Lorentz indices. The gravitational constant, $\kappa$, is related to $\alpha^{\prime}$ and the string coupling, $\lambda$, by

$$
\frac{1}{2 \kappa^{2}}=\frac{1}{(2 \pi)^{7} \alpha^{\prime 4} \lambda^{2}}
$$

We will be interested in solutions that preserve some supersymmetry. In other words the supersymmetry variation of the fermions should be zero. The fermion supersymmetry transformation laws are

$$
\begin{aligned}
\delta \chi & =F_{M N} \Gamma^{M N} \epsilon \\
\delta \lambda & =\left(\Gamma^{M} \partial_{M} \phi-\frac{1}{6} H_{M N P} \Gamma^{M N P}\right) \epsilon \\
\delta \psi_{M} & =\left(\partial_{M}+\frac{1}{4} \Omega_{-M}^{A B} \Gamma_{A B}\right) \epsilon
\end{aligned}
$$

Here $\epsilon$ is the infinitesimal supersymmetry parameter. $\epsilon$ is a Majorana-Weyl spinor of $S O(1,9)$. It has 16 real components.

Let the radius of $\mathbf{S}^{1}$ be $R$. The metric is a product of flat $\mathbf{R}^{1,5}$ and the 4 dimensional Taub-Nut metric

$$
d s^{2}=R^{2} U\left(d y-A_{i} d x^{i}\right)^{2}+U^{-1}(d \vec{x})^{2}, \quad i=1,2,3, \quad 0 \leq y \leq 2 \pi
$$

where,

$$
U=\left(1+\frac{R}{2|\vec{x}|}\right)^{-1},
$$

and $A_{i}$ is the gauge field of a monopole centered at the origin. The Taub-NUT space has the following desirable properties,

- If we excise the origin, what remains is a circle fibration over $\mathbf{R}^{3}-\{0\}$. Eq. 24) is written such that $\vec{x}$ is the coordinate on this base $\mathbf{R}^{3}-$ $\{0\}$. For $|\vec{x}|$ restricted to a constant, the fibration is exactly the Hopf fibration of $\mathbf{S}^{3}$ over $\mathbf{S}^{2}$. 
- The origin $\vec{x}=0$ is a smooth point.

- As $|\vec{x}| \rightarrow \infty$ the radius of the fiber becomes $R$.

- The space has a $U(1)$ isometry group that preserves the origin $\vec{x}=0$. An element $g(\theta)=e^{i \theta} \in U(1)$ acts by $y \rightarrow y+\theta$.

We will now show that this space with a constant dilaton and all other fields set to zero is a supersymmetry preserving solution to zero'th order in $\alpha^{\prime}$. Firstly Eq.(17) is trivially satisfied to zero'th order in $\alpha^{\prime}$. The supersymmetry variation of $\chi$ and $\lambda$ are zero since the right hand sides are zero for any $\epsilon$. The only nontrivial equation is the gravitino variation which now reads

$$
\delta \psi_{M}=\left(\partial_{M}+\frac{1}{4} \omega_{M}^{A B} \Gamma_{A B}\right) \epsilon=\nabla_{M} \epsilon
$$

where $\nabla$ is the covariant derivative in the Taub-Nut metric. Taub-Nut space is a noncompact Calabi-Yau manifold. It has $S U(2)$ holonomy, which implies that there are covariantly constant spinors of one chirality, say positive. More concretely the spin connection satisfies

$$
\omega_{M}^{A B}=\epsilon^{A B C D} \omega_{M}^{C D}
$$

where $\epsilon^{A B C D}$ is the totally antisymmetric symbol with $\epsilon^{1234}=1$. Positive chirality means that

$$
\Gamma_{1234} \epsilon_{+}=\epsilon_{+}
$$

Combining the three equations above we get

$$
\delta \psi_{M}=\partial_{M} \epsilon_{+}
$$

This is zero for a constant $\epsilon_{+}$. There are no unbroken supersymmetries of negative chirality. Since the original supersymmetry was a Majorana-Weyl spinor of positive chirality in $9+1$ dimensions the unbroken supersymmetry is a positive chirality spinor in the $5+1$ dimensions along the worldvolume of 
the brane. To higher order in $\alpha^{\prime}$ the solution changes. For instance Eq.(17) implies that $H$ is nonzero to first order in $\alpha^{\prime}$. The variation of the dilatino, $\delta \lambda$, then implies that the dilaton becomes non constant to first order in $\alpha^{\prime}$. One should imagine correcting the solution order by order in $\alpha^{\prime}$. To do that consistently we should also include higer order corrections in the original action. The point is however that there is a solution to all orders in $\alpha^{\prime}$ and the string coupling too for that sake. The exact solution will preserve supersymmetry. This is so because a BPS state comes in a small representation of the supersymmetry algebra. The size of a representation cannot jump when the parameters of the theory are changed continously. Until we have a full non perturbative formulation of string theory the only way to specify solutions is to do it in some approximation, like weak coupling. These solutions will then give rise to exact solutions.

Let us find the charges of this solution. The charges are integers and can not jump with $\alpha^{\prime}$ so we should be able to identify the charges working to lowest non-trivial order. As we saw above there are two relevant 5-brane charges, $m$ and $n$. $m$ denotes the magnetic charge of the 3 -form field,

$$
m=\frac{1}{16 \pi^{2} \alpha^{\prime}} \int_{\mathbf{S}^{2} \times \mathbf{S}^{1}} H
$$

$n$ denotes the first chern class of the circle-fibration at infinity. In this case $n=1$. What about $m$. In type II theories $m$ would be zero but it is different in the Heterotic theory due to the Bianchi identity Eq.(18). The Bianchi identity implies that the curvature induces magnetic charge of the $B$-field. We get

$$
m=\frac{1}{16 \pi^{2}} \int_{\mathbf{S}^{2} \times \mathbf{S}^{1}} \omega_{3}^{L}(\Omega)=1
$$

The higher order $\alpha^{\prime}$ corrections cannot change an integer as already noted. We thus conclude that the Taub-Nut space is the 5-brane discussed in the previous section with quantum numbers $(1,1)$. Since it preserves some supersymmetry its tension is given exactly by the formula derived in the previous 
section.

$$
T_{(1,1)}=\left|\frac{m_{s}^{6}}{\lambda^{2}}-\frac{m_{s}^{8} R^{2}}{\lambda^{2}}\right|
$$

Here $m_{s}^{2}=\frac{1}{\alpha^{\prime}}$. We see that the first term is suppressed by $\alpha^{\prime}$ compared to the second one. The second term is the ADM mass of the pure Taub-Nut solution. The first term would appear when taking the $\alpha^{\prime}$ corrections into account.

\section{The marginally stable NS5-brane}

In this section we will study the stability of the NS5-brane in the Heterotic theory on a circle. The NS5-brane is a BPS 5-brane. It has quantum numbers $(m=1, n=0)$ in the notation from section 2 . It has tension

$$
T_{(1,0)}=\frac{m_{s}^{6}}{\lambda^{2}}
$$

Since it is BPS it cannot decay into 5-branes of a total tension which is smaller than $T_{(1,0)}$. It could be however that it could split into two or more 5 -branes whose tension add up to $T_{(1,0)}$. The central charge for a 5 -brane with charges $(m, n)$ is

$$
Z_{(m, n)}=m \frac{m_{s}^{6}}{\lambda^{2}}-n \frac{m_{s}^{8} R^{2}}{\lambda^{2}}
$$

The BPS inequality reads

$$
T_{(m, n)} \geq\left|Z_{(m, n)}\right|
$$

Suppose that the NS5-brane can split into two 5-branes of charges $\left(m_{1}, n_{1}\right)$ and $\left(m_{2}, n_{2}\right)$. Then they must both be BPS and have positive central charge.

$$
\begin{aligned}
m_{1} \frac{m_{s}^{6}}{\lambda^{2}}-n_{1} \frac{m_{s}^{8} R^{2}}{\lambda^{2}} & \geq 0 \\
m_{2} \frac{m_{s}^{6}}{\lambda^{2}}-n_{2} \frac{m_{s}^{8} R^{2}}{\lambda^{2}} & \geq 0 \\
m_{1}+m_{2} & =1 \\
n_{1}+n_{2} & =0
\end{aligned}
$$


The condition that they are BPS is written down in section 2. It is that either $m$ and $n$ have opposite sign or they are both 1 or both -1 . It is easily seen that all these requirements are only satisfied if

$$
\begin{aligned}
& \left(m_{1}, n_{1}\right)=(1,1) \\
& \left(m_{2}, n_{2}\right)=(0,-1)
\end{aligned}
$$

or vice versa and furthermore

$$
m_{s} R \leq 1
$$

We thus conclude that for large radius the NS5-brane is absolutely stable as one would expect. However for a radius smaller than the selfdual radius the NS5-brane is only marginally bound. It can split into the 5-branes with charges $(1,1)$ and $(0,-1)$. The one with charge $(1,1)$ is the Taub-Nut space discussed in the previous section. The $(0,-1) 5$-brane can be thought of as an anti Taub-Nut space bound with a NS5-brane.

\section{The moduli space of Taub-Nut 5-branes}

In this section we will discuss the moduli space of several Taub-Nut 5-branes, or Kaluza-Klein monopoles, in the Heterotic theory on a circle. In section(3) we presented the supergravity solution for one Taub-Nut 5-brane. The case of $q$ parallel Taub-Nut 5-branes is similar. The metric is to lowest order the $q$-centered Taub-Nut space with metric

$$
d s^{2}=R^{2} U\left(d y-A_{i} d x^{i}\right)^{2}+U^{-1}(d \vec{x})^{2}, \quad i=1 \ldots 3, \quad 0 \leq y \leq 2 \pi .
$$

where,

$$
U=\left(1+\sum_{l=1}^{q} \frac{R}{2\left|\vec{x}-\overrightarrow{x_{l}}\right|}\right)^{-1},
$$

and $A_{i}$ is the gauge field of $q$ monopoles centered at $x_{l}, l=1, \ldots, q$. This space is smooth except when two or more centers coincide. For $n$ coinciding 
centers the space develops an $\mathbf{R}^{4} / \mathbf{Z}^{n}$ orbifold singularity. An $\mathbf{R}^{4} / \mathbf{Z}^{n}$ orbifold singularity is also sometimes denoted a $A_{n-1}$ singularity. The total charge of this system is $(q, q)$. A NS5-brane, which has charge $(1,0)$, can be thought of as a small instanton [18]. In other words a NS5-brane can spread out and become an instanton in the transverse space. The 5-branes with charge $(q, q)$ cannot spread out. Their moduli space is $4 q$ dimensional. $3 q$ of these dimensions come from specifying the $q$ centers of the $q$-centered Taub-Nut space. The last $q$ parameters come from the $B$-field. The $q$-centered TaubNut space has $q$ normalisable harmonic two-forms. The $q$ scalars come from expanding the $B$-field into these $q$ normalisable harmonic two-forms. These $q$ parameters are periodic due to gauge symmetry of the $B$-field. It is equivalent of periodicity of Wilson lines of a gauge field. 4 of these $4 q$ dimensions parametrise the center of mass motion, which is $\mathbf{R}^{3} \times \mathbf{S}^{1}$. It decouples from the rest of the moduli space.

The moduli space of $q$ Kaluza-Klein monopoles has been argued by Sen to be equal to the moduli space of $q$ BPS monopoles in four-dimensional $\mathcal{N}=4$ $S U(2)$ gauge theory [19]. For coinciding 5 -branes the space has an $A_{q-1}$ singularity. The moduli space of the Heterotic theory on a $A_{q-1}$ singularity has recently been studied by Witten [6]. He conjectured that it is equal to the moduli space of 3-dimensional $\mathcal{N}=4$ pure gauge theory with gauge group $S U(q)$. Similar statements were conjectured for $D$ and $E$ groups. In the case of $q=2$ it was shown explicitly that the moduli space is equal to the Atiyah-Hitchin space. Shortly after, Witten's conjecture was proven by means of string dualities [7, 8, 9].

The purpose of this section is to prove that the moduli-space of the twocentered Taub-Nut space is equal to the Atiyah-Hitchin space. In other words the moduli space does not depend on the radius $R$ of the circle at infinity. This result follows from Sen's identification of this moduli space with a monopole moduli space, but here we will prove it with a different argument. We will show this by following Witten's approach [6] and see that 
the argument used in the case of an $A_{1}$ singularity also goes through in the case of two-centered Taub-Nut space.

Firstly the moduli space is a hyper-Kähler manifold. This is because it is the moduli space in a 6 -dimensionsonal theory with 8 supercharges without gravity. There is no gravity because the transverse space is noncompact the 6-dimensional gravitational constant is zero. Hence the moduli space is that of a globally supersymmetric theory, i.e. hyper-Kähler.

The moduli space is independent of the string coupling constant, since it is a hypermultiplet moduli space. By further compactifying to 4 dimensions the string coupling sits in a vector multiplet moduli space and hence does not correct the hypermultiplet moduli space [20]. It can hence be studied in Heterotic conformal theory.

Discarding the center of mass motion the moduli space is 4 dimensional. When the 2 5-branes are far away from each other the moduli space is parametrised by the separation, $\vec{x}$, and the integral of the $B$-field along the exceptional divisor. To lowest order in $\alpha^{\prime}$ the moduli space is just $\left(\mathbf{R}^{3} \times \mathbf{S}^{1}\right) / \mathbf{Z}_{2}$. The $\mathbf{Z}_{2}$ is due to the indistinguishability of the 2 branes. Interchanging them changes the orientation on the exceptional divisor and hence changes the sign of the integral of the $B$-field.

In the case of an $A_{1}$ singularity Witten showed that $\alpha^{\prime}$ corrections change the topological structure of the moduli space at infinity. The circle is not in a product with $\mathbf{R}^{3}$ at infinity but is in a nontrivial circle bundle of first Chern class -4 . Exactly the same is true here as we will now argue. The actual Heterotic spacetime is of course different in this case compared to the case of a pure singularity but the circle in the moduli space behaves similarly.

When the two 5-branes are on top of each other, the Taub-Nut space has an exact $U(1) \times S U(2)$ symmetry. The $U(1)$ tranalates along the circle in Taub-Nut space. The $S U(2)$ rotates the $\mathbf{R}^{3}$. When the two 5-branes are separated the $S U(2)$ acts on their separation vector. So we have an exact $S U(2)$ that acts on the moduli space. The $U(1)$ does not act on the 
moduli space. In the case of the $A_{1}$ singularity there was a $\operatorname{Spin}(4)=$ $S U(2)_{L} \times S U(2)_{R}$ which acted on the space. The $S U(2)_{R}$ acts on the moduli space whereas the $S U(2)_{L}$ does not. The important point is that in our case we do still have a $S U(2)$ that acts on the moduli space. At infinity the moduli space is approximately $\left(\mathbf{R}^{3} \times \mathbf{S}^{1}\right) / \mathbf{Z}_{2}$. The $S U(2)$ rotates the $\mathbf{R}^{3}$. The question of what bundle the circle is in over the $\mathbf{R}^{3}$ at infinity is the same as asking how the $S U(2)$ acts on the circle. Let us fix a specific point, $\vec{x}$, in $\mathbf{R}^{3}$. There is a $U(1)$ subgroup of $S U(2)$ which leaves this point invariant. This $U(1)$ will act on the $\mathbf{S}^{1}$ with a charge $k$. This $k$ is exactly the first chern class of the bundle. Hence we have to determine $k$. $k$ can be determined in the same way as in the case of the pure $A_{1}$ singularity [6]. Here we will consider a worldsheet instanton, i.e. a fundametal Euclidean string wrapped on the exceptional divisor. The partition function of this string should be invariant under the $U(1)$ which leaves $\vec{x}$ invariant. There are two potential non-invariances of this partition function. One comes from the path integral over worldsheet fermions which gives a factor of the Pfaffian of the worldsheet Dirac operator, $\operatorname{Pf}(\mathcal{D})$. The other is the coupling of the $B$-field to the fundamental string $\exp \left(i \int B\right)$. The product of the two

$$
\operatorname{Pf}(\mathcal{D}) \exp \left(i \int B\right)
$$

should be invariant under $U(1)$. Performing a $U(1)$ transformation $e^{i \alpha}$ the factor $\exp \left(i \int B\right)$ get multiplied by $e^{i k \alpha}$, where $k$ is the first Chern class which we want to find. $\operatorname{Pf}(\mathcal{D})$ gets multiplied with $e^{i n \alpha}$ where $n$ is the total $U(1)$ charge of the fermionic zero modes of the Dirac operator. For this to be invariant we must have $k=-n$. Hence we just have to count the total charge of the fermionic zero modes. In the case of the pure $A_{1}$ Witten counted this to be 4 . The point is now that the presence of the circle cannot change this since it is an integer. More precisely, let $R>>|\vec{x}|>>\sqrt{\alpha^{\prime}}$. Then the situation is almost indistinguishable to the case of a pure singularity and $n=4$. Now deform $R$ continously to any value. $n$ stays an integer and hence 
$n=4$.

Now we have established that the moduli space at infinity is a circle fibration over $\mathbf{R}^{3}$ with first chern class -4 and there is a $S U(2)$ symmetry that acts on the moduli space with generic three-dimensional orbits. This space should be modded out by $\mathbf{Z}_{2}$. The moduli space is also smooth by the argument in [6]. There is no $U(1)$ that acts on the fiber at infinity. It is broken by worldsheet instanton effects. As noted above the moduli space is hyper-Kähler. The point is now that there is a unique space with these properties, namely the Atiyah-Hitchin space [21]. We thus conclude that the moduli space of 2 Kaluza-Klein 5-branes is the Atiyah-Hitchin space.

\section{Acknowledgements}

This work was supported by the National Science Foundation grant PHY 94-07194. 


\section{References}

[1] D.J. Gross, J. Harvey, E. Martinec and R. Rohm, "Heterotic string", Phys. Rev. Lett. 54 (1985) 502

[2] A. Font, L. Ibanez, D. Lust and F. Qeevedo, Phys. Lett. B249 (1990) 35

[3] A. Sen, "Strong-Weak Coupling Duality in Four Dimensional String Theory", Int.J.Mod.Phys.A9:3707-3750,1994, hep-th/9402002

[4] A. Sen, " AN INTRODUCTION TO NONPERTURBATIVE STRING THEORY", hep-th/9802051

[5] C.M. Hull and P.K. Townsend, Unity of Superstring Dualities", Nucl. Phys. B438 (1995) 109-137, hep-th/9410167

[6] E. Witten, "Heterotic String Conformal Field Theory And A-D-E Singularities", hep-th/9909229

[7] M. Rozali, "Hypermultiplet Moduli Space and Three Dimensional Gauge Theories", hep-th/9910238

[8] P.S. Aspinwall and M.R. Plesser, "Heterotic String Corrections from the Dual Type II String", hep-th/9910248

[9] P. Mayr, "Conformal Field Theories on K3 and Three-dimensional Gauge Theories", hep-th/9910268

[10] P. Horava and E. Witten, "Heterotic and Type I String Dynamics from Eleven Dimensions", Nucl. Phys. B460 (1996) 506-524, hep-th/9510209

[11] P. Ginsparg, Phys. Rev. D35 (1987) 648

[12] T. Banks, M. Dine, H. Dijkstra and W. Fischler, Phys. Lett. B212 (1988) 45 
[13] J. Harvey and J. Liu, Phys. Lett. B268 (1991) 40

[14] R. Khuri, Phys. Lett. B259 (1991) 261, Phys. Lett. B294 (1992) 325, Nucl. Phys. B387 (1992) 315

[15] J. Gauntlett, J. Harvey and J. Liu, Nucl. Phys. B409 (1993) 363

[16] C.G. Callan, J.A. Harvey and A. Strominger, "Supersymmetric String Solitons", Lectures at the 1991 Trieste Spring School on String Theory and Quantum Gravity, hep-th/9112030

[17] M. Green and J. Schwarz, Phys. Lett. B151 (1985) 21

[18] E. Witten, "Small Instantons in String Theory", Nucl. Phys. B460 (1996) 541, hep-th/9511030

[19] A. Sen, "Dynamics of Multiple Kaluza-Klein Monopoles in M- and String Theory", Adv. Theor. Math. Phys. 1 (1998) 115-126, hep-th/9707042

[20] B. de Wit, P. Lauwers and A. Van Proeyen, "Lagrangians of $\mathrm{N}=2$ Supergravity-Matter Systems", Nucl. Phys. B255 (1985) 269

[21] M.F. Atiyah and N. Hitchin, "The Geometry And Dynamics Of Magnetic Monopoles", Princeton University Press, 1988 